\title{
Changing Trends in Biliary Stenting for Unresectable Malignant Perihilar Obstructions
}

\author{
Lubna Kamani' and Muhammad Arshad ${ }^{2}$ \\ ${ }^{1}$ Department of Gastroenterology, Liaquat National Hospital and Aga Khan University Hospital, Karachi, ${ }^{2}$ Department of Surgery, Aga \\ Khan University Hospital, Karachi, Pakistan
}

See "Inside Plastic Stents versus Metal Stents for Treating Unresectable Malignant Perihilar Biliary Obstructions: A Retrospective Comparative Study” by Yoshihide Kanno, Shinsuke Koshita, Takahisa Ogawa, et al., on page 735-742.

Endoscopic biliary stenting for the management of benign and malignant strictures is the first choice across the globe to relieve obstruction and cholangitis. Since there are two types of stents used, plastic and self-expandable metal stents (SEMS), clinical efficacy of one over the other is a much debated topic in the medical literature. A meta-analysis by Zorrón Pu et al. was in favor of SEMS, in terms of longer stent patency time and better survival with lower rates of re-interventions, compared to plastic stents in malignant perihilar obstructions. ${ }^{1}$ The use of inside plastic stents (iPS) is relatively a new approach in the palliative management of malignant hilar strictures where a stent is placed in the suprapapillary position with a nylon thread attached at the distal end of the stent for easy removal of stent later on. Recently, in a few Japanese studies, iPS have been tested in a single arm study ${ }^{2,3}$ and compared with transpapillary plastic stents (TPS) and SEMS with mixed results. ${ }^{4}$ These studies are mainly single center studies with a small sample size. Inatomi et al. did not find any statistical difference in the median patency period between the iPS and SEMS groups $(p=0.83) .{ }^{4}$ The main hypothesis is that iPS prevents

Received: June 18, 2020 Revised: July 12, 2020

Accepted: July 13, 2020

Correspondence: Lubna Kamani

Department of Gastroenterology, Liaquat National Hospital and Aga Khan University Hospital, National Stadium Road, Karachi 74800, Pakistan Tel: +92-30-02562141, Fax: +92-21-34140014, E-mail: lkamani@yahoo.com ORCID: https://orcid.org/0000-0003-2651-5179

(cc) This is an Open Access article distributed under the terms of the Creative Commons Attribution Non-Commercial License (http://creativecommons.org/ licenses/by-nc/3.0) which permits unrestricted non-commercial use, distribution, and reproduction in any medium, provided the original work is properly cited. reflux of duodenal secretions, bacteria, and food that results in early stent blockage ${ }^{5}$ as compared to TPS. There is a dearth of literature regarding the comparison of iPS and SEMS in terms of clinical effectiveness, complication rates, and stent patency period.

In this issue of Clinical Endoscopy, Kanno et al. compared the clinical efficacy, complications, and recurrent biliary obstruction (RBO) outcome of iPS with SEMS in patients with unresectable perihilar malignant obstruction. ${ }^{6}$ They showed that although there were no differences in clinical effectiveness and complications between the two groups, interestingly the median time to RBO was prolonged in the iPS group compared to SEMS $(p=0.008)$. Despite having its own limitations of being a single center, retrospective study with limited patients enrolled, especially in iPS group, it is overall a well-structured manuscript. Inculcating the concept of the usage of plastic stents in hilar obstruction, which can later be removed and replaced with new stents, is fascinating and could open new avenues for future treatments of malignant hilar biliary strictures, keeping aside the traditional approach of using SEMS in these patients. They have reported a complication rate of $8 \%$ including pancreatitis, acute cholangitis, and death in SEMS group. The authors have not discussed reasons for complications in this group, especially the need for sphincterotomy, difficulty in stent placement, or selection of stents at the time of placement.

Baseline characteristics of the two groups in this study were similar to some extent, but in the SEMS group there were more patients with Bismuth level IV strictures with solid irregularity on surface images and more patients with cholangitis 
(though statistically not significant), which could be reasons for the short median time to RBO in this group. Furthermore, the type, size, placement technique of SEMS (side by side vs. partial stent in stent), and number of stents to be placed was at the discretion of the performing endoscopist, which can lead to selection bias. It would also be interesting to know the stage of the disease, co-morbidities, and functional class of these patients as this may affect the overall outcome of the stent patency period. There was no overall improvement in survival between the two groups, except for those who received chemotherapy in which survival was significantly improved, whereas randomized controlled trial (RCT) conducted by Sangchan et al. revealed a significant survival benefit in their SEMS group. ${ }^{\text {? }}$ There were no procedure related complications in the plastic group in this study, but a study by Koizumi et al. reported that there is an increased risk of stone formation in inside stents. ${ }^{8}$ In a RCT study conducted in Denmark by Pedersen et al., it was concluded that there was increased stent migration among the stents placed above the sphincter of Oddi compared to the transpapillary approach $(p=0.026)$, whereas no difference in overall stent performance was noted among the two groups. ${ }^{9}$ On the contrary, a Chinese study by Yan et al. highlighted that over length suspended plastic stents have improved patency periods compared to conventional plastic stents..$^{10}$ Again, this study was retrospective and single center based with a limited number of patients and the results cannot be generalized.

Deploying multiple iPS compared to multiple SEMS for palliation of malignant biliary stricture seems cost effective, especially from a developing country perspective, although in a meta-analysis ${ }^{1}$ they failed to show any statistically significant cost difference between these two groups. This finding needs to be validated in an adequately designed cost effective analysis study.

The survival of patients with pancreaticobiliary cancer is likely to increase with further advancements in medical management, multidisciplinary approaches, and improved palliative care. Hence, the need to maintain biliary flow for prolonged periods will be required in the future to provide an improved quality of life to patients with unresectable malignant biliary obstructions. Since, at present, we do not have an ideal biliary stent which does not block, the need for stent replacements will remain.

The idea of keeping both ends of the stent inside the biliary system is appealing as it prevents the exposure of one end to the bowel lumen contents, which predisposes it to obstruction and infection. The feasibility of iPS replacement adds to its advantage. Other specialties have long moved in this direction, especially urology where percutaneous or transurethral placement of double J stents with both ends of stent inside the urinary system is the gold standard to provide drainage for an obstructed kidney.

In conclusion, the idea of using suprapapillary plastic stent insertion in non-operable malignant hilar stricture is still evolving and its effectiveness over SEMS needs more validation. In order to make iPS accepted as a standard of care, adequately powered studies in the form of multi-center RCT, ideally with three arms (iPS, transduodenal stenting, and SEMS) along with eliminating confounding factors is recommended.

\section{Conflicts of Interest}

The authors have no financial conflicts of interest.

Author Contributions

Conceptualization: Lubna Kamani

Resources: LK

Supervision: Muhammad Arshad

Writing-original draft: LK

Writing-review\&editing: MA

ORCID

Muhammad Arshad: https://orcid.org/0000-0002-1681-2025

\section{REFERENCES}

1. Zorrón Pu L, de Moura EG, Bernardo WM, et al. Endoscopic stenting for inoperable malignant biliary obstruction: a systematic review and meta-analysis. World J Gastroenterol 2015;21:13374-13385.

2. Kaneko T, Sugimori K, Shimizu Y, et al. Efficacy of plastic stent placement inside bile ducts for the treatment of unresectable malignant hilar obstruction (with videos). J Hepatobiliary Pancreat Sci 2014;21:349-355.

3. Ishiwatari H, Hayashi T, Ono M, Sato T, Kato J. Newly designed plastic stent for endoscopic placement above the sphincter of Oddi in patients with malignant hilar biliary obstruction. Dig Endosc 2013;25 Suppl 2:94-99.

4. Inatomi O, Bamba S, Shioya $\mathrm{M}$, et al. Threaded biliary inside stents are a safe and effective therapeutic option in cases of malignant hilar obstruction. BMC Gastroenterol 2013;13:31.

5. van Berkel AM, van Marle J, Groen AK, Bruno MJ. Mechanisms of biliary stent clogging: confocal laser scanning and scanning electron microscopy. Endoscopy 2005;37:729-734.

6. Kanno Y, Koshita S, Ogawa T, et al. Inside plastic stents versus metal stents for treating unresectable malignant perihilar biliary obstructions: a retrospective comparative study. Clin Endosc 2020;53:735-742.

7. Sangchan A, Kongkasame W, Pugkhem A, Jenwitheesuk K, Mairiang P. Efficacy of metal and plastic stents in unresectable complex hilar cholangiocarcinoma: a randomized controlled trial. Gastrointest Endosc 2012;76:93-99.

8. Koizumi M, Kumagi T, Kuroda T, et al. Endoscopic stent placement above the sphincter of Oddi for biliary strictures after living donor liver transplantation. BMC Gastroenterol 2020;20:92.

9. Pedersen FM, Lassen AT, Schaffalitzky de Muckadell OB. Randomized trial of stent placed above and across the sphincter of Oddi in malignant bile duct obstruction. Gastrointest Endosc 1998;48:574-579.

10. Yan X, Huang Y, Chang H, Zhang Y, Yao W, Li K. Suspended over length biliary stents versus conventional plastic biliary stents for the treatment of biliary stricture: a retrospective single-center study. Medicine (Baltimore) 2018;97:e13312. 